\title{
Cuatro argumentos sobre el concepto de intervención social
}

\author{
FOUR ARGUMENTS ABOUT THE CONCEPT OF SOCIAL INTERVENTION
}

Dr. Juan Saavedra (jsaavedr@ubiobio.cl) Departamento Ciencias Sociales, Universidad del Bío-Bío (Concepción, Chile)

\begin{abstract}
This article exposes four arguments on the debate about the nature and conceptual range of social intervention, understanding it equally as action, interpretation, distinction and finally as a discursive dispositive. This debate has relevance for social sciences disciplines in which intervention notion has an important place in its theoretic-methodological structure. This review sets a counterpoint with technological perspectives that reduce intervention to action's execution. In this way, there is a discussion about epistemic and ontological social sciences communities' subordination in which intervention is a hallmark.
\end{abstract}

Key words: social intervention, applied epistemology, arguments, complexity, discourse.

\section{Resumen}

En este artículo se presentan cuatro argumentos expresados en el debate sobre la naturaleza y alcance conceptual de la intervención social, entendiéndola indistintamente como actuación, interpretación, distinción sistémica y finalmente como dispositivo discursivo. Este debate es relevante para disciplinas de las ciencias sociales en las que la noción de intervención ocupa un lugar importante en su configuración teórico-metodológica. La revisión de estas perspectivas establece un contrapunto con las perspectivas tecnológicas que sostienen que la intervención se reduce a la ejecución de acciones. De este modo, se discute la subordinación epistémica y ontológica de las comunidades de las ciencias sociales, en las cuales intervenir constituye su sello de identidad.

Palabras clave: intervención social, epistemología aplicada, argumentación, complejidad, discurso.

\section{Introducción}

En la actualidad en diversos campos de las ciencias sociales se discute sobre los alcances teóricos, epistemológicos y éticos del concepto de intervención social, siendo sus aspectos prácticos los que han sido objeto de mayor atención en las últimas décadas. Así, por ejemplo, la psicología social establece sobre este concepto una vía para emplear sus perspectivas teóricas propias, las cuales aportarán desde este campo disciplinario a la resolución efectiva de problemas sociales. Por su parte, en el trabajo social discurre una aceptación más bien identitaria en torno a la noción de intervención, que opera como una suerte de sello distintivo de su ejercicio profesional, el cual también es recogido, entre otros documentos, por la definición del concepto de trabajo social realizada por la International Federation of Social Workers (IFSW) en la Conferencia de Montreal del año 2000. Con todo, siguiendo autores como 
Ezequiel Ander-Egg, Nidia Aylwin, Natalio Kisnerman, entre otros, en el caso particular del trabajo social es importante destacar que la intervención social se concibe tradicionalmente como una actividad práctica propia de esta profesión.

A pesar de lo anterior, en las últimas décadas surgen nuevas perspectivas que expresan puntos de vistas diferentes sobre la intervención, en los que se exhortan otros razonamientos teóricos que admiten posicionamientos novedosos, sobre la base de convicciones emergentes en torno a la naturaleza del concepto. Estas perspectivas recogen distintas formas en las cuales se define la intervención social, de cuya lectura es posible distinguir sus usos en varios de los campos disciplinarios de las ciencias sociales en los que la noción es referida. Se aprecia que en este debate los encasillamientos de los autores y sus perspectivas se centran en un conjunto más bien definido de hilos comunes que conectan dichas proposiciones y que, a nuestro juicio, se dirigen a constituirse en cuatro núcleos de argumentaciones sobre la intervención social.

\section{Argumentación como epistemología aplicada}

Apoyarse en la noción de argumentación, en vez de otras acepciones tales como corrientes o enfoques, es relevante para distinguir ópticas diversas sobre el asunto de la intervención social. Esta opción no es neutra, pues implicará asentar una forma de aproximación epistemológica aplicada en los términos expuestos por Santibáñez (2012). La argumentación, en su sentido más generalizado, designa una actividad discursiva en la cual se busca persuadir o convencer. Para Vicuña (2004), la argumentación supera la tradicional búsqueda de la verdad en la construcción de la argumentación, para situarse en lo que esta autora denomina como el ideal de razonabilidad propuesto por la dialéctica de Van Eemeren y Grootendorst, según la cual quien busca persuadir evita pronunciarse sobre la cuestión de la verdad. Más bien, la perspectiva dialéctica de la argumentación busca concebir su sustentabilidad en los razonamientos, lógicas y marcos filosóficos que se esbozan para la resolución de una disputa o debate. Concordando con Warley, tras "toda teoría científica se encuentra una cierta filosofía que la orienta y, en última instancia, la define" (2004:328). Con todo, en la lectura de los autores de la intervención social se evidencia que existe una interesante discusión en este campo, la que desde luego abarca a quienes defienden la centralidad disciplinaria de esta noción versus quienes critican el fundamento teórico y ético de su utilización. Así, por ejemplo, las opiniones de Olga Vélez y Pablo Suárez son críticas al uso del concepto en el marco del trabajo social.

Entonces, reconociendo que el uso del término intervención social está legitimado por un conjunto de disciplinas de las ciencias sociales, es importante aclarar que no existe unanimidad sobre su conceptualización. El concepto de intervención es objeto de una controversia significativa sobre su naturaleza y alcance conceptual, por lo cual el examen de las posiciones argumentales resulta ilustrativo de la forma en que es configurada teórica y metodológicamente en la actualidad. Volviendo a la posición de Santibáñez, quien recoge postulados de la obra de Toulmin de 1958, los argumentos conjugan una dinámica organicista para sostener que, en campos como las ciencias sociales, las estructuraciones de conocimiento "justifican sus pretensiones y respaldan sus juicios" (Santibáñez 2012:32). En este sentido, lo que está detrás de las posiciones sobre la intervención no sólo es la divergencia epistémica, sino que la posibilidad de influir en los encasillamientos teórico-metodológicos de las comunidades disciplinarias que resignifican la intervención social en sus prácticas y marcos teóricos.

Los estudios sobre la argumentación en el campo de las ciencias han generado creativas soluciones para abordar las nuevas perspectivas sobre un determinado problema o interés disciplinario en las ciencias 
sociales (como sucede con la intervención). Ciapuscio resalta la capacidad heurística de los usos del lenguaje en la construcción conceptual e interpretativa, lo cual contribuye a la formulación de figuras de encuadre de las diversas perspectivas en grupos de argumentaciones. Este camino, señalado primariamente desde la epistemología aplicada de Toulmin, recorre el camino de la argumentación como una epistemología que involucra conocimientos, acuerdos y cambios conceptuales. Desde esta perspectiva, las argumentaciones en la intervención social apuntan a sostener ciertas premisas que refuerzan las posiciones en el debate teórico.

A nuestro juicio las principales líneas argumentativas localizan a la intervención social en: a) el ámbito de la actuación o acción de ciertas profesiones, b) como una forma de interpretación de la complejidad social, c) la que por extensión también aplica a la distinción funcional de los sistemas sociales y que finalmente se expresa en d) los términos de dispositivos de intervención. A continuación, profundizaremos en cada una de estas argumentaciones.

\section{La intervención social como acción práctica}

Para esta argumentación, la intervención constituye una forma de actuar de una categoría de profesiones del mundo social, orientada a la resolución de problemas sociales. Desde este punto de vista, la intervención es conceptualizada como una forma de actividad que integra aspectos políticos, filosóficos y procedimentales, evocando la idea de kinesis. Este hacer está vinculado con saberes teóricos y técnicos, pero especialmente con actitudes, valores y creencias que anteponen eticidad a la acción. En este sentido, Ander-Egg señala que la intervención social designa "el conjunto de actividades realizadas de manera más o menos sistemática y organizada, para actuar sobre un aspecto de la realidad social con el propósito de producir un impacto determinado" (1995:161). Desde la óptica de este autor, la intervención social es tributaria de las perspectivas psicosociales que en Europa visibilizan el problema de la acción técnica-profesional en la sociedad, tomándose como ejemplo la definición de la intervención elaborada por el Colegio de Psicólogos Sociales de España de 1984, según la cual corresponde a una actividad profesional que surge como respuesta a la necesidad de analizar y actuar sobre los problemas de las interacciones personales en sus diversos contextos sociales.

En general, la argumentación práctica de la intervención se enfoca en el carácter organizado de la acción y su capacidad para resolver problemas sociales. Tributaria de esta argumentación está la opinión de Fernando Fantova, quien señala que la intervención social es una actividad que intenta responder a necesidades sociales, siendo su propósito la integración, autonomía, bienestar y participación de las personas en relación a su entorno. En este mismo sentido, Ferrán Casas-Aznar considera que la acción de intervenir tiene su inicio en situaciones consideradas como negativas, riesgosas o que constituyen aspiraciones de mejoramiento de una comunidad determinada.

Otro autor que transita por esta línea argumental es Javier Corvalán, quien define la intervención social como: "la acción organizada de un conjunto de individuos frente a problemáticas sociales no resueltas en la sociedad a partir de la dinámica de base de la misma. Esta dinámica de base [...] es por un lado, el funcionamiento capitalista en torno al sistema de mercado que determina cualitativa $y$ cuantitativamente la producción de bienes y servicios, y por otra parte, el derecho público y privado que regula tanto la apropiación legítima de tal producción por parte de los individuos como los conflictos de intereses entre los mismos" (1997:4). En esta definición se aprecia que el autor comparte con Ander-Egg el carácter organizado de la intervención social, no obstante, vincula el ethos de estas prácticas con el funcionamiento de las economías de mercado y las sociedades que articulan su vida en torno a cierta 
disposición del consumo de bienes y servicios. En nuestra opinión, el concepto de Corvalán discurre por un camino de mayor reconocimiento de las estructuras socioeconómicas evidentes en las últimas décadas, incorporando una perspectiva de los derechos de los sujetos en marcos de conflictos de interés, en los cuales es necesario actuar desde las instituciones para procurar la resolución de los problemas sociales: este sería el escenario de la práctica en el que se despliega la intervención social.

Es así que desde la visión de Corvalán coexisten dos tipos de intervención social: a) la caritativa o asistencial y b) la socio-política, esta última relacionada con la forma de implantación del modelo de desarrollo de corte capitalista. Esta perspectiva sociopolítica de la intervención está asociada al proceso de modernidad en cuanto al desarrollo del pensamiento crítico, la práctica democrática, las consecuencias de la revolución industrial y la creciente secularización de la cultura occidental.

Desde España, Esperanza Molleda (2007) se pregunta por qué no es factible el hacer intervención, atendiendo a las condiciones de trabajo y la urgencia del actuar, que imposibilitan el seguimiento de los protocolos de actuación pre-establecidos en las instituciones que implementan servicios sociales. Molleda introduce un aspecto relevante en esta línea argumental de la acción, localizando el problema de la intervención en la sujeción a la razón instrumental, que padece de un exceso de formalización. Parafraseando las posturas teóricas de Horkheimer, Molleda pretende criticar la desustancialización de la práctica, para acotarla a la mera razón funcional de medios. La autora propone redefinir la intervención social con un fuerte anclaje teórico y ético identitario, señalando que este tipo de actuación corresponde a las actividades o acciones que se realizan de manera formal u organizada, respondiendo a las necesidades sociales con el propósito tanto de prevenir, paliar o corregir procesos de exclusión social, como promover procesos de inclusión o participación social.

\section{La intervención social como interpretación de la complejidad social}

Una segunda línea de argumentación sostiene que la intervención es antes que todo una interpretación de la complejidad de lo social. En otras palabras, hay intervención social desde el momento en que se interpreta la complejidad del entramado que manifiesta un ámbito conflictivo o problemático de lo social. En esta perspectiva, la intervención es concebida desde una relación dialógica, en la cual la aproximación tanto hermenéutica como a la vez compleja a los fenómenos sociales es la adecuada para interpretar los ámbitos de expresión de los problemas sociales, teniendo en cuenta que para ello el "fenómeno social se comprende de entrada como complejo, y por consiguiente, no simple y llanamente como un agregado de partes" (Maldonado 2011:149). La intervención social emerge como acto de atribución de sentido que supone un proceso de aproximación a los contextos, narraciones y testimonios de la situación. También exige descartar la asepsia valorativa del mundo, más bien, esta argumentación acepta que en la intervención existen preconfiguraciones de sentido desde los operadores de las políticas sociales, los cuales complejizan tanto la explicación de los fenómenos sociales como el modo en que se actúa sobre estos problemas.

En relación a la interpretación, es necesario indicar que sus fuentes más confiables están localizadas en el campo de la hermenéutica, que gracias al llamado giro lingüístico de las ciencias sociales transita efectivamente desde el campo de la filología a sus aplicaciones en el estudio de la sociedad. Habermas indica que la hermenéutica está relacionada a la capacidad interpretativa que se adquiere junto al dominio de la lengua materna, y en este sentido, la idea de la interpretación refiere a despejar aquello que aparece como confuso o poco claro, y es en este aclarar-sentido que se asocia al acto del comprender. No obstante, las posibilidades de la aclaración interpretativa están paradojalmente 
relacionada con las implicancias de la complejización de la hermenéutica de lo social, incluyendo en el acto de comprender aquello que está interrelacionado con su contexto, y por consiguiente, excluyendo del campo de comprensión los simples objetos aislados de su entorno. La concepción de un pensamiento complejo, en los términos declarados por Edgar Morin, no puede sustraerse de las implicancias en la interpretación de las relaciones entre conceptos, actantes y significados del mundo social que constituyen las contingencias propias de la intervención.

Existen además indicaciones recurrentes hacia la perspectiva de la complejidad de Morín en esta argumentación sobre la intervención social, contando con algunos autores que suscriben a este enfoque. Así por ejemplo, Auxiliadora González y Germán Jaraíz (2013) definen la intervención social como una mediación intencionada que ordena un proceso de acción en torno a la relación compleja de tres dimensiones de lo social (bienestar social, calidad de vida y desarrollo social), para lo cual los autores señalan que la intervención debe ser comprendida como un hecho social complejo. Para esto, proponen establecer un diálogo combinado entre las tres lógicas de intervención que sostienen en esta conceptualización, que a nuestro juicio, es consistente con una perspectiva que releva la interpretación sobre los contextos y marcos de producción de sentidos, sobre los cuales, se constituyen las expresiones de la intervención social (instituciones, servicios sociales, programas).

En este horizonte de complementariedad interpretación y complejidad, localizamos las propuestas teóricas de Teresa Matus, quien a partir de la concepción de la polifonía busca explicar la ruptura de las ópticas de mundo moderno en los que se presenta la intervención social. En este sentido, la autora sostiene la necesidad de aprender a reconstruir los mecanismos de interpretación polifónica de la complejidad social. La perspectiva supone que "es posible pensar en alternativas, reasumiendo una relación contradictoria de teoría y praxis en el horizonte de una comprensión social compleja, de una intervención social fundada en otros parámetros" (Matus 2005:14). En esta perspectiva, Matus define la intervención social como un proceso de categorización simbólica, que contribuye a la producción de subjetividades y la constitución identitaria, en el sentido que "no hay intervención sin interpretación social" (Matus 2002:27). Como consecuencia de lo anterior, la autora señala que en los procesos de intervención social no se trabaja con individuos en cuantos tales, sino que más bien con categorías analíticas. De este modo, la intervención distingue múltiples subjetividades específicas (ej. mujeres trabajadoras, jóvenes infractores de ley, entre otras) y no recurre a contenedores genéricos utilizados tradicionalmente en el trabajo social, como son el caso social, el grupo y la comunidad. Para esta argumentación, es necesario superar la comprensión estática del actante de la intervención, para constituirse primero en intérprete de la compleja red de significados que configuran un problema social.

Compresión compleja quiere apuntar a la articulación significativa de las trasformaciones contextuales, la teoría social, los enfoques epistemológicos y las perspectivas éticas concurrente, dando sentido a la idea de aproximaciones múltiples a los problemas sociales objetos de la intervención. En una alusión indirecta a la polifonía de Matus, Gianinna Muñoz sugiere la idea del contrapunto, "que consiste en el arte de combinar, según reglas, las diferentes voces presentes en un mismo espacio" (2011:93), lo que en otros términos permite adecuar la interpretación compleja en la intervención social. Esto, a partir de la concepción de observadores que aprecian lo social desde su particularidad disciplinaria, pero que simultáneamente distinguen desde la complementariedad dialógica y las posibilidades de la traducción hermenéutica que representa la transdiciplinariedad. En los términos señalados por Muñoz, la síntesis en la que podemos localizar esta línea de argumentación permite que la intervención social esclarezca significados, busque acuerdos en los desacuerdos interpretativos y concrete acuerdos operativos sobre las actuaciones concurrentes. 


\section{La intervención social como distinción de los sistemas sociales funcionales}

Esta tercera línea argumental también aparece vinculada a la idea de la complejidad, pero desde un contenido que no siempre es concordante con los principios hermenéuticos revisados anteriormente. Desde este punto de vista, la teoría social introduce la noción de sistema social complejo, que opera a partir de sus distinciones, lo que implica que "la posibilidad de dibujar esta distinción surge de las descripciones que auto-elabora la sociedad en su propio proceso de producción" (Santibáñez 1997:116). Esta línea de pensamiento, incluida en el marco de la teoría de sistemas sociales, corresponde a una tradición de la cual Niklas Luhmann es uno de sus principales exponentes.

La idea de la complejidad sistémica no puede ser únicamente representada por la cantidad de elementos y relaciones identificables en un sistema. En la lógica de Luhmann, la relación entre el sistema y el entorno se caracteriza por la diferencia de grados de complejidad, de tal modo que sus múltiples alternativas conducen a que los sistemas deban actuar selectivamente con estas, o dicho de otra forma, deban constantemente reducir su complejidad.

En esta secuencia argumental, nos interesa presentar algunos de los postulados propuestos por Fernando Robles a propósito del trabajo social, pero que son extensibles a la intervención como tal. Robles (2002) señala que en los sistemas sociales la operación más genuina está radicada en la comunicación, y como tal, se conecta a otras operaciones de los sistemas sin necesariamente aludir a los sistemas de consciencia de los cuales está desacoplada. En términos de la intervención social, sólo es posible efectuarla en los ámbitos de lo comunicable, pero no lo es en lo referente a lo que los sujetos piensan o sienten, pues estas operaciones están contenidas en el ámbito de los sistemas de la conciencia (psiquis).

Para Robles, en el funcionamiento de los sistemas sociales parciales, operan reglas de inclusión/exclusión a partir de procesos de selección, mediante códigos específicos (de carácter binario) que regulan la pertenencia o no a las operaciones de dichos sistemas. Así, por ejemplo, en el sistema parcial del mercado, la inclusión/exclusión es determinada por la operación pagar/no pagar. No obstante, en el caso de trabajo social -en cuanto sistema especializado en intervención- suceden dos paradojas relevantes. La primera, es que a diferencia de los demás sistemas parciales que tienden a la exclusión de las comunicaciones ajenas a sus operaciones, se incluye a las comunicaciones excluidas de otros sistemas parciales. El trabajo social tiene por objeto reparar las comunicaciones dañadas y consecuentemente, opera sus propios mecanismos de exclusión en la formula incluir para excluir. La segunda paradoja está dada por la operación interna del sistema trabajo social que interviene fuera de los límites del mismo. En los términos de Robles, "el sistema del trabajo social no significa que los casos estén allá afuera, él los construye internamente, según la medida de los criterios propios que constituyen su programación y usando prestaciones de otros sistemas de la sociedad como el derecho, la economía o la política" (2002:18).

En otro hilo de esta argumentación sobre la intervención social, a propósito de la planificación social, Harold Dupuis señala que la intervención proyectada permite coordinar la variabilidad de los sistemas con las posibilidades selectivas de las mismas, de modo tal que los procesos evolutivos sociales adquieran reflexividad. Para el autor, una intervención con carácter sistémico permite transformaciones en sociedades altamente diferenciadas. A propósito del estudio de esta categoría de sociedades, Aldo Mascareño indica que la intervención social "solo es posible como orientación sistémica contextual, es decir, como una invitación e incentivo a la autorregulación de sistemas (sociales y psíquicos) autónomos, 
operativamente clausurados y cognitivamente abiertos a su entorno sobre la base de sus propias distinciones" (Mascareño 2011:2). En este sentido, en los procesos de intervención radican las capacidades de selección comunicativa de los sistemas y en su viabilidad autorregulada.

El enfoque de sistemas sustenta consistentemente este hilo de argumentación en torno a la intervención aludiendo a la capacidad de los individuos para situarse en el entorno de la sociedad, por lo cual "conservan una autonomía relativa que les permite evaluar, sin idealizaciones, sus posibilidades concretas de influir en ella" (Dockendorff 2013:158). La prolongación de esta argumentación basada en la perspectiva sistémica funcional-estructuralista paulatinamente está encontrando mayor resonancia en el diseño de algunas de las políticas públicas implementadas en Chile de las últimas décadas, especialmente en los ámbitos del trabajo con familias y potenciación del desarrollo territorial. Con ello, la extensión de esta lógica argumentativa posibilita el contraste con las miradas asentadas en el positivismo, las cuales dominaron tanto las vertientes conceptuales como las aproximaciones metodológicas de la intervención social hasta bien avanzado el siglo XX.

\section{La intervención social como dispositivo discursivo}

La cuarta argumentación en torno a la intervención se localiza en el campo de la discursividad. Calsamiglia y Tuson señalan que el discurso es esencialmente una práctica social que refiere a "una forma de acción entre las personas que se articula a partir del uso lingüístico contextualizado" (2008:1) sea este oral o escrito. Wodak señala que el discurso es "un complejo conjunto de actos lingüísticos simultáneos y secuencialmente interrelacionados" (2003:105) que se manifiesta en forma oral y escrita. Desde esta perspectiva, la intervención social puede ser comprendida en el campo discursivo, no sólo por los actos orales de habla (ej. los mensajes presidenciales), sino que también en los textos, que constituyen productos duraderos de estas acciones lingüísticas. En este sentido, las enunciaciones de las políticas sociales, como los fundamentos de programas, proyectos y servicios sociales representan el ámbito de configuración discursiva de la intervención.

En este sentido, la evolución de los estudios de discurso permite la convergencia entre las ciencias sociales y los enfoques lingüísticos del mismo, los que serán útiles para efectos de localizar a la intervención social en este ámbito. Inicialmente los estudios lingüísticos se dirigían a indagar el alcance de las normas gramaticales que lo rigen, pero a partir de inicios del siglo $X X$, toman un nuevo rumbo que va a impactar no sólo en el surgimiento de una ciencia del texto, sino que en todo el amplio espectro de la filosofía, las humanidades y las ciencias sociales. En este giro lingüístico, entre otros, es relevante el trabajo de Michel Foucault y su supuesto inicial relativo al discurso: "Yo supongo que en toda sociedad la producción del discurso está a la vez controlada, seleccionada y redistribuida por un cierto número de procedimientos que tienen por función conjurar los poderes y peligros, dominar el acontecimiento aleatorio y esquivar su pesada y temible materialidad" (Foucault 1992:5).

El discurso es la expresión del poder, con capacidad de movilizar no sólo la voluntad y los cuerpos de los sujetos, sino que también de incidir en la generación de nuevos discursos articulados o no, que a su vez se instituyen como otras expresiones de poder. El repertorio discursivo de la intervención se ha renovado en diversas líneas de argumentación. Entre estas encontramos el trabajo de Alfredo Carballeda, quien define la intervención social como "un conjunto de dispositivos de asistencia y de seguros en función de mantener el orden o la cohesión de lo que denominamos sociedad" (2002:91). Desde la perspectiva argumental del autor, la intervención social es una artificialidad configurada en un lugar y tiempo determinado, para dar cuenta de las consecuencias activas de la cuestión social, en 
cuanto expresión del malestar de vastos colectivos humanos que experimentan los trastornos derivados de la instauración del capitalismo como matriz ordenadora de la vida social, cultural y económica desde fines de siglo XVIII. La nueva cuestión social extiende sus consecuencias a los ámbitos privados de la existencia, y resignifica los lugares en los que se expresa. El lugar de la intervención se configura en lo que Foucault denomina un territorio (donde se ejerce el poder), es decir, "un espacio jurídico, que habla de la legitimidad de la intervención, y político, que marca la agenda donde se construyen diferentes aspectos de la cuestión social" (Carballeda 2002:95). El ejercicio del poder para asegurar el orden social releva además la condicionante normativizadora en el discurso de la intervención, en cuanto la vida social sigue un modelo biológico como curso regulador (Muhle 2009). Esta traza biológica de la regulación se acopla a las geografías, los cuerpos y las instituciones, de modo tal que "en la intervención social radica la regulación de la conducta de la población, en función a lo que es considerado como normal y como se generan dispositivos con propósitos de contensión o de estimulación, según sea el caso" (Saavedra 2015:32).

La posición discursiva ofrece un punto de vista particular que permite tejer un argumento respecto a la intervención social, basado fundamentalmente en la teoría del poder de Foucault, y que es posible relacionar con el carácter fundado de la intervención social. Coincidiendo con Carballeda, la intervención está irrefutablemente ligada a los idearios de la modernidad y al proceso de desplazamiento reflexivo que experimenta la humanidad en los últimos cinco siglos. Para que esto tenga sentido, las hipótesis foucaultianas son útiles para entender que en las sociedades de control, el poder se vuelve más humano y cotidiano, y en su puesta en escena, los procesos de intervención son claves para disciplinar a quienes son cooptados por los idearios, beneficios y claves conceptuales de la modernidad capitalista. Dicho de otro modo, la intervención social se materializa en un conjunto de políticas, programas y servicios enmarcados no sólo en los productos/beneficios que oferta a la población, sino que también en un discurso por el cual "se regula intencionadamente la cuestión social, en sus fronteras e implicancias" (Saavedra 2015:142).

El origen de la intervención es localizado en la configuración temprana de la modernidad, precisamente en el pensamiento de Hobbes y su concepción contractualista de la sociedad. El pacto de sujeción que transfiere al soberano la libertad natural de la humanidad, constituye un principio para la paz, pero además funda las bases de la cohesión para lograrla. La intervención social expresa el discurso de lo deseable y de lo no-deseable (anormalidad) en la configuración de la sociedad. En la necesidad de establecer los perfiles de dichas transgresión, la anormalidad inicialmente fue vista como una expresión demoníaca que es susceptible de ser tipologizada y ordenada, tal como describe Foucault. En esta pugna entre lo natural y lo sobrenatural, comienza a visibilizarse en los albores de la modernidad una forma primigenia de la filantropía, que paulatinamente reemplazará al orden pastoral cristiano, que en términos de Foucault, son la base del poder predisciplinario. Este desplazamiento hacia una posición laica de la intervención no implicará un cambio de sentido paradigmático en el discurso sobre la intervención social, sino más bien, la coexistencia con una postura basada en la caridad, que es posible distinguir inclusive en la actualidad.

El Estado es clave en la irrupción del laicismo en la intervención social, y es quien se propone dirigir la conducta humana en función de enunciados ideales que expresan la normalidad en la sociedad. Así, desde el Estado se definirá el discurso que regulará el tipo de familia esperado, el ejercicio de la maternidad o paternidad, la forma de relación entre los sujetos y el Estado, entre otros preceptos. Para este efecto, nuevamente se configurará un discurso sobre nuevos sujetos, que en un sentido derridiano, dejará escritura (y sus huellas) en los informes sociales, las pautas de entrevista, notas de derivación y la 
formulación de programas de intervención social. El disciplinamiento de conductas consideradas como disfuncionales, antisociales, desviadas o problemáticas comienza a configurar al sujeto de la intervención social en los márgenes del proyecto moderno: emerge un consistente discurso sobre la pobreza, sus implicaciones éticas y mecanismos de tratamiento-contención. En este punto se introduce en este argumento discursivo el carácter político en la configuración de la intervención social. En efecto, tras la instauración de la figura del Estado nacional, la intervención se localiza en el área enunciativa de las políticas y programas sociales, que distingue la anormalidad en vastos sectores de la población pauperizada, los que requieren del orden moderno operado en forma de ayuda o beneficencia pública.

Desde la vereda de la historia social, María Angélica Illanes (2006) también poner referencia en este caso propone una serie de categorías conceptuales que guían la construcción del discurso de la intervención en Chile. Así, a diferencia de la narrativa histórica más clásica de la cuestión social, Illanes se sitúa desde la incorporación institucional del pueblo al proyecto de modernidad, reconociendo una forma de materialidad cultural que esta autora ha denominado como el cuerpo y la sangre del pueblo. Esta categoría es relevante, pues entre otras, reconoce en las primeras visitadoras sociales (década de 1930) una suerte de prédica de un nuevo pacto social entre el pueblo y el nuevo Señor representado ahora por el Estado. En esta categoría se visualiza de mejor forma el disciplinamiento corporal implícito en la intervención social, idea que nos vincula con esta perspectiva más discursiva del fenómeno.

Es entonces que la representación de la intervención social como un dispositivo -en la lógica de Foucault- resulta útil para la comprensión del argumento discursivo, pues sitúa su configuración en la forma en que los hablantes son capaces de reconocer formas disciplinarias en las heterogeneidades propias de los dispositivos sociales. Con todo, la tarea de desenmarañar el concepto de dispositivo en la obra de Foucault no resulta del todo realizable. Edgardo Castro (2009) nos propone las siguientes consideraciones para aproximarnos al concepto: a) los dispositivos constituyen una red de relaciones de elementos heterogéneos, b) establecen al mismo tiempo las condiciones para abordar la naturaleza del vínculo de relación entre las heterogeneidades, c) los dispositivos son estratégicos, pues responden a la necesidad de urgencia en lo social (control del delito, absorción de inmigrantes, violencia escolar, etc.), d) además de definirse por sus elementos heterogéneos, los dispositivos se delimitan por las circunstancias históricas de su génesis, y por último, e) que una vez constituidos como tales, sus efectos entran en juego de resonancia o contradicción con otros dispositivos, lo que exige su continuo ajuste a las condiciones históricas de su reconocimiento.

La formulación de los dispositivos de intervención busca incorporar en esta línea de argumentación la posibilidad de aprehender el carácter político de este fenómeno, utilizándolo como un recurso interpretativo pero también operacional de la gubernamentalidad. Con todo, los debates epistemológicos en torno a la noción de dispositivo son conceptual y metodológicamente intricados, atendiendo a las continuas observaciones que el propio Foucault hace del concepto, como también de las exégesis posteriores realizadas por variados autores, como por ejemplo, por Deleuze. En el caso de la intervención social, la vía de argumentación discursiva sostiene las posibilidades de distribuir los saberes que operan desde el poder y que se expresan en los mecanismos de aproximación a la cuestión social, como sucede con los programas gubernamentales extendidos, por ejemplo, sobre la población más pobre. 


\section{Síntesis}

En la figura 1 se sintetizan las formas de argumentación revisadas en este artículo, relativas a la conceptualización de la intervención social. Esta matriz propuesta toma como punto de partida el núcleo enunciativo de cada una de las cuatro afirmaciones argumentales (B) que encuadran la forma de aplicación a la que suscribe dicha perspectiva (C), y la cual, se sostiene en base de antecedentes epistemológicos delimitados tanto por la tradición como por la emergencia de perspectivas no convencionalistas (A). Esta síntesis expresa la posibilidad de madurar una línea de pensamiento que se construye sobre la revisión de una epistemología aplicada a la articulación de estas perspectivas teóricas sobre la intervención social. Este ejercicio, no sólo involucra una mirada asertiva sobre la naturaleza conceptual de la propia intervención, sino de un diálogo entre los fundamentos y el método, o en otras palabras, entre la filosofía y la aplicabilidad de las ciencias sociales en torno a situaciones específicas que son observadas desde la intervención social.

Las potencialidades de las argumentaciones como epistemología aplicada resultan útiles para lograr un encuadre apreciativo de las diversas perspectivas conceptuales que rondan en torno a la idea de la intervención social: como actuación, interpretación, distinción sistémica y finalmente como dispositivo discursivo. Aún más, la revisión de estas perspectivas adiciona un debate post-argumental, en donde el problema de la intervención social tiene necesariamente un correlato epistemológico que trasciende la mera practicidad, pese a las consideraciones tecnológicas que (auto)subordinaron históricamente a las comunidades del campo de las ciencias sociales en las cuales el intervenir constituye su eje de su formulación teórico-metodológico.

Fig.1. Síntesis de las perspectivas argumentativas sobre la intervención social (elaboración propia)

\begin{tabular}{|c|c|c|c|}
\hline $\begin{array}{c}\text { A } \\
\text { Antecedente }\end{array}$ & $\begin{array}{c}\text { B } \\
\text { Afirmación }\end{array}$ & \multirow{5}{*}{$\begin{array}{l}\text { Por } \\
\text { tanto, se } \\
\text { expresa } \\
\text { en }\end{array}$} & $\begin{array}{c}\text { C } \\
\text { Aplicación }\end{array}$ \\
\hline $\begin{array}{l}\text { Tradición de } \\
\text { corte } \\
\text { positivista }\end{array}$ & $\begin{array}{l}\text { La intervención } \\
\text { social es actividad o } \\
\text { kinesis }\end{array}$ & & $\begin{array}{l}\text { Ejecución de } \\
\text { acciones } \\
\text { configuradas en } \\
\text { planes-proyectos }\end{array}$ \\
\hline $\begin{array}{l}\text { Perspectiva de } \\
\text { la complejidad }\end{array}$ & $\begin{array}{l}\text { La intervención } \\
\text { social es } \\
\text { interpretación } \\
\text { compleja }\end{array}$ & & $\begin{array}{l}\text { Interpretación de } \\
\text { subjetividad }\end{array}$ \\
\hline $\begin{array}{l}\text { Enfoque } \\
\text { funcional- } \\
\text { sistémico }\end{array}$ & $\begin{array}{l}\text { La intervención } \\
\text { social es distinción } \\
\text { de sistema parcial }\end{array}$ & & $\begin{array}{l}\text { Distinción de } \\
\text { inclusión/exclusión }\end{array}$ \\
\hline $\begin{array}{l}\text { Enfoque } \\
\text { discursivo/ } \\
\text { post } \\
\text { estructural }\end{array}$ & $\begin{array}{l}\text { La intervención } \\
\text { social es discurso } \\
\text { (dispositivo) }\end{array}$ & & $\begin{array}{l}\text { Enunciación- } \\
\text { configuración de } \\
\text { dispositivos }\end{array}$ \\
\hline
\end{tabular}

Finalmente, concordando con el punto de vista de Ana María Vicuña sobre las posibilidades heurísticas de la argumentación, que reconoce las discusiones sobre un determinado tema, ésta confiere a las disciplinas de las ciencias sociales centralmente involucradas en el estudio y aplicación de la intervención social (trabajo social y psicología social principalmente) una interesante oportunidad para revisar las 
convicciones teóricas y epistemológicas que están tras dichas argumentaciones. Esto permite apreciar la riqueza de un debate en pleno desarrollo en las primeras décadas este siglo XXI. Así, sostener que la intervención se traduce en una forma de actuación, implica asentar sus métodos y prácticas derivadas en los principios epistemológicos propios de la hegemonía positivista en las ciencias sociales de la primera mitad del siglo XX y, por otra parte, cuando se entiende que este fenómeno es más complejo, es porque atiende a los postulados de las corrientes sistémicas o post-estructuralistas que sostienen dichas argumentaciones, de cuyo examen es posible deslindar conceptos como la noción de dispositivo discursivo, en cuanto entidad heterónoma de regulación biopolítica. Y cuando referimos a esa distinción heterónoma, estamos refiriendo a que la naturaleza de los componentes del dispositivo son diversos (legislación, arquitecturas, sujetos, agentes, entre otros), todos los cuales paradojalmente, configuran una unidad homogénea de normativización que regula diversos aspectos de la vida de la población. Pensar en enfoques epistémicos emergentes permiten dinamizan este debate, de modo tal que la discusión conceptual de la intervención social contemporánea entronca su curso en una variedad de posibilidades, recusaciones y convergencias.

\section{Bibliografía}

Ander Egg, E. 1995. Diccionario del trabajo social. Buenos Aires: Lumen.

Carballeda, A. 2002. La intervención en lo social: exclusión e integración en los nuevos escenarios sociales. Buenos Aires: Paidos.

Casalmiglia, H. y Tuson, A. 2008. Las cosas del decir. Manual de análisis del discurso. Barcelona: Ariel.

Castro, E. 2009. El vocabulario de Michel Foucault. La Plata: Universidad Nacional de la Plata.

Corvalán, J. 1997. Los paradigmas de lo social y las concepciones de intervención en la sociedad. Santiago: CPU.

Dockendorff, C. 2013. Antihumanismo o autonomía del individuo ante las estructuras sociales. Cinta moebio 48: 158-173. doi: 10.4067/S0717-554X2013000300004

Foucault, M. 1992. El orden del discurso. Buenos Aires: Tusquets.

González, A. y Jaraiz, G. 2013. La intervención social: una mirada desde esquemas de complejidad. En: E. Ballesteros y J. Solana. Complejidad y ciencias sociales. Sevilla: U.I. Andalucía, pp. 263-293.

Illanes, A. 2006. Cuerpo y sangre de la política social. La construcción histórica de las visitadoras sociales 1887-1940. Santiago: LOM.

Maldonado, C.E. 2011. Complejidad de los sistemas sociales: un reto para las ciencias sociales. Cinta moebio 36: 146-157. doi: 10.4067/S0717-554X2009000300001

Mascareño, A. 2011: Sociología de la intervención: orientación sistémica contextual. Revista Mad 25: 133. doi: 10.5354/0718-0527.2011.15656 
Matus, T. 2002. Propuestas contemporáneas en trabajo social. Para una intervención polifónica. Buenos Aires: Espacio.

Matus, T. 2005. Apuntes sobre intervención social. Santiago: PUC.

Molleda, E. 2007. ¿Por qué decimos que "no podemos hacer intervención social"? Cuadernos de Trabajo Social 20: 139-155. http://revistas.ucm.es/index.php/CUTS/article/view/CUTS0707110139A

Muhle, M. 2009. Sobre la vitalidad del poder: una genealogía de la biopolítica a partir de Foucault y Canguilhem. Revista de ciencia política (Santiago) 29(1): 143-163. doi: 10.4067/S0718090X2009000100008

Muñoz, G. 2011. Contrapuntos epistemológicos para intervenir lo social: ¿cómo impulsar un diálogo interdisciplinar? Cinta moebio 40: 84-104. doi: 10.4067/S0717-554X2011000100005

Robles, F. 2002. Opciones de reinclusión para domiciliaridades dañadas. Espacio Abierto 11(1): 9-24.

Saavedra, J. 2015. Liturgia de la intervención social. Discursos presidenciales en tiempos del neoliberalismo 1981-2015. Concepción: Andén Sur.

Santibáñez, D. 1997. Investigación social y autorreferencia. Cinta moebio 2: 114-128. http://www.cintademoebio.uchile.cl/index.php/CDM/article/viewFile/26477/27771

Santibáñez, C. 2012. Teoría de la argumentación como epistemología aplicada. Cinta moebio 43: 24-39. 10.4067/S0717-554X2012000100003

Vicuña, A. 2004. Los criterios de aceptabilidad en el discurso argumentativo. En: A. Harvey. En torno al discurso. Contribuciones de América Latina. Santiago: Ediciones PUC, pp. 46-55.

Warley, J. 2004. Epistemología, argumentación y polifonía. Anclajes 8(8): 323-341.

http://ojs.fchst.unlpam.edu.ar/ojs/index.php/anclajes/article/view/329

Wodak, R. 2003. Métodos de análisis crítico del discurso. Barcelona: Gedisa.

Recibido el 27 Oct 2014

Aceptado el 3 Ene 2015 